\title{
Spectral characteristics of composite obtained by embedding of magnetic nanoparticles into polymer matrix
}

\author{
I. V. Pleshakov ${ }^{1}$, A. V. Prokof'ev ${ }^{1}$, E. E. Bibik ${ }^{2}$, E. K. Nepomnyashchaya ${ }^{3}$, \\ E. N. Velichko ${ }^{3}$, T. A. Kostitsyna ${ }^{3}$, D. M. Seliutin ${ }^{3}$ \\ ${ }^{1}$ Ioffe Institute, 26 Politechnicheskaya str., Saint Petersburg, 194021, Russia \\ ${ }^{2}$ Saint Petersburg State Institute of Technology (Technical University), 26 Moskovsky ave., \\ 190013, Saint Petersburg, Russia \\ ${ }^{3}$ Peter the Great Saint Petersburg Polytechnic University, 29 Polytechnicheskaya str., \\ 195251, Saint Petersburg, Russia \\ ivanple@yandex.ru
}

PACS 42.70.-a; 42.70.Jk; 75.50.Mm;

DOI 10.17586/2220-8054-2021-12-3-279-282

\begin{abstract}
The transmission spectra of a composite material obtained by introducing magnetite nanoparticles into a polyvinyl alcohol matrix are studied. The samples were films on the glass substrate prepared by drying an aqueous solution of polymer and a ferrofluid. A number of them were made in a constant magnetic field, which led to the formation of extended aggregates. The features of these spectra and possible causes of their appearance are discussed.
\end{abstract}

Keywords: nanocomposite, magnetite nanoparticles, ferrofluid, polyvinyl alcohol.

Received: 13 May 2021

Revised: 24 May 2021

\section{Introduction}

A composite, which is a polymer matrix with a nanodispersed filler, is a topic of interest both for basic science and for a wide variety of applications [1]. It is expected that among the plenty of such structures, in those where the integrated phase is a magnetically ordered compound, additional new properties, for example, the possibility to control their characteristics by an external field, may appear [2].

One of the simplest and most promising ways to produce a compound of this type is to prepare it from a mixture of a ferrofluid and a matrix-forming polymer dissolved in the same medium. This approach was used in the present work, where, on the basis of polyvinyl alcohol (PVA), the film samples with nanoparticles of magnetite $\left(\mathrm{Fe}_{3} \mathrm{O}_{4}\right)$ were fabricated from an aqueous ferrofluid (FF) and PVA solution. These specimens, being transparent, were an optical material and their spectral properties were the subject of our study.

\section{Experiment}

\subsection{Samples}

The FF we used was a water-based magnetite colloid (the methods for producing such substances and their main properties are described in [3]). Its stabilization, i.e., preventing the nanoparticles adhesion, was performed by creating a double layer on the surface of $\mathrm{Fe}_{3} \mathrm{O}_{4}$, consisting of molecules with hydrophobic (inner) and hydrophilic (outer) portions, which are oleic acid salts. The diameter of the magnetite core was approximately $10 \mathrm{~nm}$, and the total diameter of the particle together with the shell was about $15 \mathrm{~nm}$ (which is slightly larger than in those FFs where a single layer of surfactant is used). The concentration of the solid phase in the liquid was about 2 vol. \%.

PVA solution was made by dissolution of its dry powder in deionized water (5 wt. $\%$ of PVA to water weight) at the temperature of $90^{\circ} \mathrm{C}$. It was filtered through a cotton and then diluted with the heated to the same temperature $\mathrm{FF}$ in a ratio of $1: 4$ (FF volume to PVA solution volume). The mixture was thoroughly stirred and kept at $90^{\circ} \mathrm{C}$ for about an hour. Then it was left for two days in a hermetically sealed vessel, preventing evaporation. The resulting substance was a homogeneous liquid.

The samples were created by applying a drop of solution to a glass substrate, which, during drying at room temperature, formed a film with a thickness of 30-35 microns. In the same way, control samples of pure PVA films were made. The films with FF were dried both without a field and in a magnetic field with strength of $\mathrm{H}=5 \mathrm{kOe}$ applied in the film plane (for five hours). Visually, the samples differed: in the first case, they were a homogeneous, 
fairly transparent material of a brownish color, and in the second - a material with a pronounced structure in the form of thin filamentous formations oriented along the field and separated by almost transparent areas.

Micrographs of the samples are shown in Fig. 1a depicts that, despite the external homogeneity, the composite prepared without a field comprises the droplets with the sizes of the order of ten micrometers. It is known that large aggregates of magnetic nanoparticles are formed in FF with certain additives [4-6], and, as it follows from our experiment, an aqueous solution of PVA produces the same effect. In the field, the agglomerates stretch out, forming a system of elongated objects, Fig. 1b. Their dimensions in thickness are tens of microns, which nearly corresponds to the data of other studies [5]. Note that along with the large prolate structural elements in Fig. 1b, the smaller ones are clearly visible, the precursors of which were, apparently, smaller droplets.
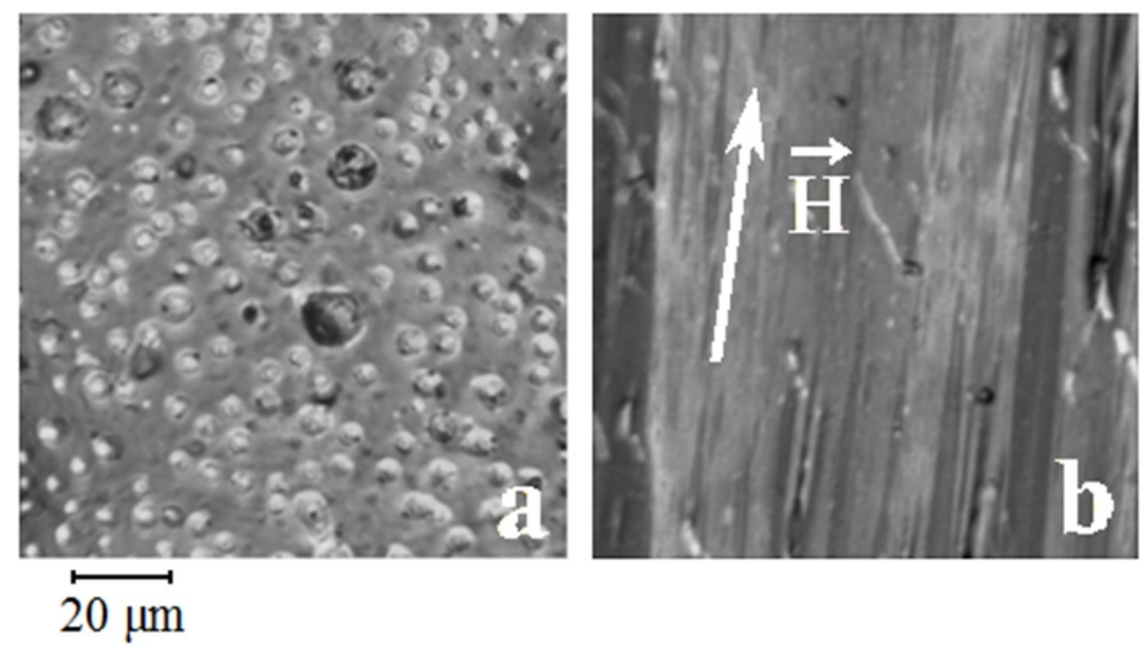

FIG. 1. Micrographs obtained for the samples prepared without field (a) and in the field $\mathrm{H}=5 \mathrm{kOe}(\mathrm{b})$

To compare the experimental results obtained on the samples described above with the spectrum of a "pure" magnetic material in the solid phase, film obtained by drying highly concentrated (18 vol. $\left.\% \mathrm{Fe}_{3} \mathrm{O}_{4}\right) \mathrm{FF}$ on glass was also prepared.

\subsection{Measurements}

In our experiments we measured transmission spectra of the films in the wavelength $(\lambda)$ range of $250-850 \mathrm{~nm}$ by a setup that included broadband light source L10290 (Hamamatsu) with deuterium and halogen lamps and spectrometer C10083MD (Hamamatsu) to detect the transmitted optical radiation. To apply the light to the sample, an optical fiber was used. The instrument function of the system was taken into account when processing the spectra.

The results of measurements on the composite materials were compared with the data obtained for pure PVA films on glass.

\section{Results}

Repeated experiments on recording the transmittance $\mathrm{T}$ of samples prepared at different times, carried out at different input optical radiation powers, gave qualitatively similar results. Figs. 1,2 exhibit typical data identifying the presence of peculiarity in the spectral characteristics of our composites.

Figure 2 shows the spectrum $\mathrm{T}(\lambda)$ of the film obtained without the application of an external field, which is compared with the same characteristic of the FF in the liquid state. To measure the latter, a standard cuvette filled with a low-concentration ( 0.02 vol. \% solid phase content) FF was used. A noticeable difference in these curves is apparent: in the liquid phase, the usual monotonic decrease in $\mathrm{T}$ in the range of $700-330 \mathrm{~nm}$ is observed (as an example, the similar data for kerosene-based FF can be found in [7]), in contrast to which the film with particle inclusions shows a significant increase in $\mathrm{T}$ at $\lambda<450-500 \mathrm{~nm}$. The sample obtained by drying of FF on a glass substrate did not display this feature. So, the behavior of the film spectrum must be related to the properties of the composite structure.

Figure 3 shows the transmission spectra of samples made with and without magnetic field (Fig. 3a), and also represents the spectrum of a sample with a pure PVA film (Fig. 3b). In general, the transmission of the film produced at the field action, increased (as expected due to the presence of transparent areas), while maintaining the feature of "enlarged translucence" at low $\lambda$. This may be perceived as the presence of a certain "peak", but a comparison of the 


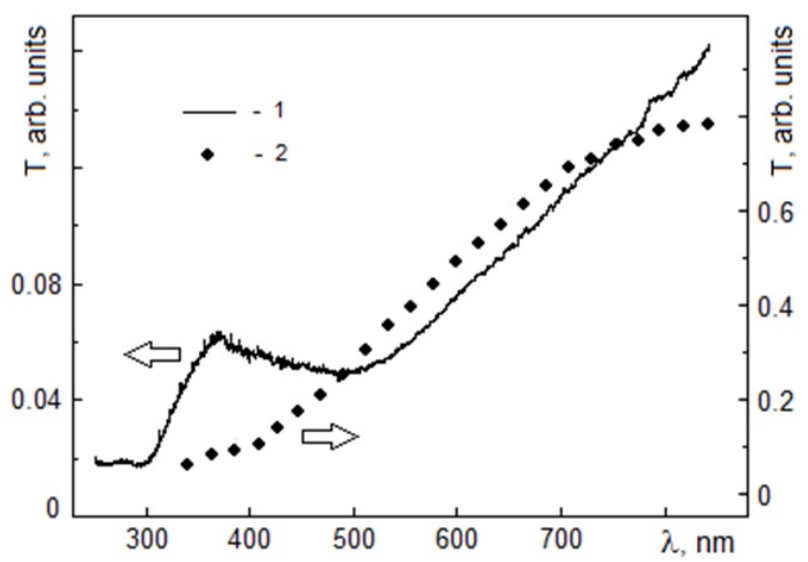

FIG. 2. Transmittance of the film prepared without field (1) compared with the transmittance of the liquid FF sample with solid phase concentration of $0.02 \%$ (2)

data for composite films with $\mathrm{T}(\lambda)$ in Fig. 3b shows that the glass with the PVA film acts as a kind of filter that cuts off light with $\lambda<350 \mathrm{~nm}$. Thus, the corresponding curves may actually continue to increase at smaller wavelengths. However, the effect of material translucence at $\lambda$ less than $450 \mathrm{~nm}$ remains unquestionable.

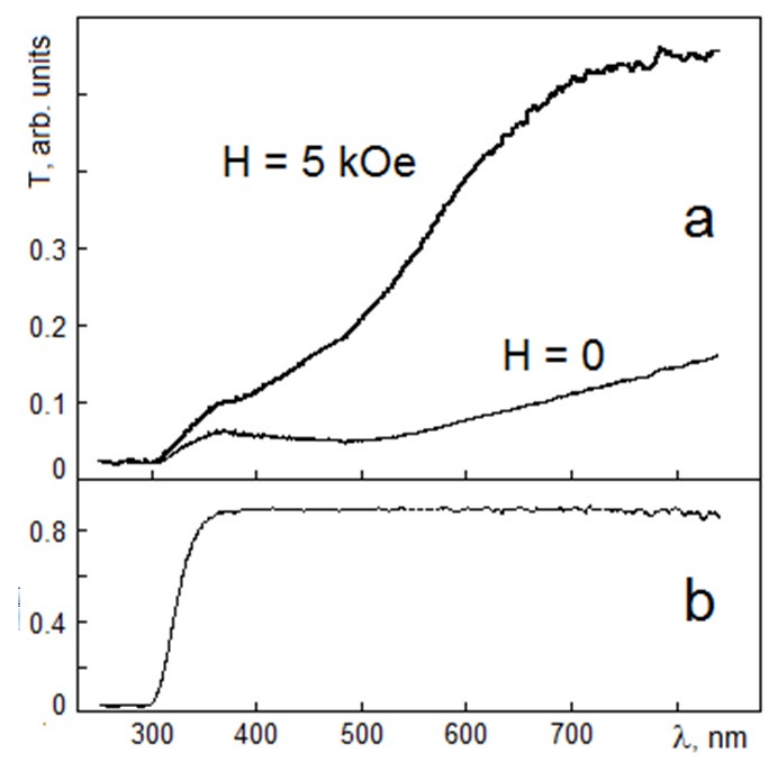

FIG. 3. Spectral characteristics of different samples: a - transmittance of the films prepared without magnetic field and in the field $\mathrm{H}=5 \mathrm{kOe} ; \mathrm{b}$ - transmittance of the film of pure PVA

\section{Discussion}

The light transmission of the FF solution is determined by processes of scattering and absorption on small particles of the material of solid phase. To separate these mechanisms may be difficult, but it is obvious that the smaller the $\lambda$, the greater the role of Rayleigh scattering on objects with a diameter of $\mathrm{d} \sim 10 \mathrm{~nm}$, i.e., those that mainly make up the FF (the larger clusters also may exist in the solution, but their number can be regarded as insignificant [8]). The application of a magnetic field to such a medium leads to the formation of agglomerates, which affects its optical properties [9].

In our case, as shown in Fig. 1a, even a sample prepared in the absence of a magnetic field already contains large (D 10 $\mu \mathrm{m}$ ) droplet formations, which radically changes the scattering. Although scattering does not stop at $\mathrm{D} \gg \lambda$, it takes on a different character [10]. Moreover, the number of scattering centers becomes many orders of magnitude smaller (the upper limit of it can be roughly estimated, assuming that all individual particles have entered to the drops, 
and, consequently, the number of scatterers have become smaller by (D/d) ${ }^{3} \sim 10^{9}$ times). Drops in this situation will rather create a shadow effect. In fact, the part of the extinction that is associated with scattering turns out to be significantly weakened, which leads to the translucence of the sample in this range.

Samples manufactured in the magnetic field are much more transparent than those that were not exposed to the field (Fig. 3a), which is associated with a decrease in the shadow effect and with a decrease in the density of structural elements (Fig. 1b). An increase in the transmittance at $\lambda<450 \mathrm{~nm}$ was also observed here, which can presumably be explained by an additional decrease in the number of small scattering centers included in the extended magnetic nanoparticle aggregates formed by the field.

\section{Conclusion}

Thus, we performed a spectroscopic study of a nanostructured material containing magnetic particles. It is shown that a fundamental role in the analysis of the optical characteristics of this composite is played by taking into account the change in light scattering associated with both the peculiarities of the incorporation of nanoparticles into the polymer matrix and with the action of a magnetic field during sample preparation.

\section{References}

[1] Crosby A.J., Lee J.Y. Polymer nanocomposites: the "nano" effect on mechanical properties. Polym. Rev., 2007, 47(2), P. 217-229.

[2] McHenry M.E., Laughlin D.E. Nano-scale materials development for future magnetic applications. Acta Mater., 2000, 1, P. $223-238$.

[3] Scherer C., Figueiredo Neto A.M. Ferrofluids: Properties and applications. Braz. J. Phys., 2005, 35(3A), P. 718-727.

[4] Zakinyan A.R., Dikansky Yu.I. Effect of microdrops deformation on electrical and rheological properties of magneticfluid emulsion. J. Magn. Magn. Mat., 2017, 431, P. 103-106.

[5] Prokof'ev A.V., Pleshakov I.V., Bibik E.E., Kuz'min Yu.I. An optical investigation of the geometric characteristics of aggregates formed by particles of magnetic fluid. Tech. Phys. Lett., 2017, 43(2), P. 194-196.

[6] Shlyagin M.G., Agruzov P.M., Pleshakov I.V., Prokofiev A.V., Bibik E.E. Incident-power-dependent refractive index of ferrofluid in magnetic field measured with a fiber optic probe. Optik, 2019, 186, P. 418-422.

[7] Hoffmann B., Köhler W. Reversible light-induced cluster formation of magnetic colloids. J. Magn. Magn. Mat., 2003, 262(2), P. 289-293.

[8] Nepomnyashchaya E.K., Prokofiev A.V., Velichko E.N., Pleshakov I.V., Kuzmin Yu.I. Investigation of magneto-optical properties of ferrofluids by laser light scattering techniques. J. Magn. Magn. Mat., 2017, 431, P. 24-26.

[9] Mohapatra D.K., Philip J. Investigations on magnetic field induced optical transparency in magnetic nanofluids. Opt. Mater., 2018, 76, P. 97105.

[10] Bohren C.F., Huffman D.R. Absorption and scattering of light by small particles. John Wiley \& Sons, Inc., NY, 1998, 544 p. 